\title{
Estado del arte y tendencias futuras en ingeniería inversa
}

\author{
V. Borja-Ramírez, R. Bell , M. López-Parra, S.D. Santillán-Gutiérrez y L.A. González-González \\ Centro de Diseño y Manufactura, Facultad de Ingeniería, UNAM y \\ Department of Manufacturing Engineering, Loughborough University, Reino Unido \\ Email:vicenteb@servidor.unam.mx
}

(recibido: mayo de 1998; aceptado: agosto de 1998)

\begin{abstract}
Resumen
En tiempos recientes, la ingeniería inversa se ha convertido en un tema relevante para industriales y académicos. Unos ven en ella sistemas compuestos por equipos y software útiles para reproducir componentes, mientras otros plantean grandes retos para desarrollar teorías de modelado y tecnologías para captura e interpretación de información. Aquí se presentan diversas interpretaciones de la ingeniería inversa, se hace un resumen sobre las principales líneas de investigación y se abordan las metodologías más avanzadas que se exploran actualmente. Con base en la información presentada, los autores expresan su opinión respecto al futuro de la investigación y aplicaciones de la ingeniería inversa.
\end{abstract}

\section{Abstract}

Nowadays, reverse engineering has become an important issue for industry and academia. The former sees reverse engineering as a system integrated by hardware and software aimed at reproducing components, whilst the other sets up research lines for the development of new models and technologies to retrieve and interpret product information. This paper presents representative points of view on reverse engineering, summarises the main research streams on the area and introduces to the more advanced methodologies which are currently explored. In the conclusions of the paper, which are based on the information presented, the authors express their opinions on future research and applications of reverse engineering.

\section{Acrónimos}

CAD Del inglés Computer Aided Design. Diseño asistido por computadora.

CAE Del inglés Computer Aided Engineering. Ingeniería asistida por computadora.

CAM Del inglés Computer Aided Manufacture. Manufactura asistida por computadora.

\section{Introducción}

El término ingeniería inversa es utilizado en diversas áreas de la ingeniería para denotar una variedad de métodos, técnicas y resultados. Las interpretaciones del término empleadas en ingeniería en computación (West, 1993) y electrónica (Rauch, 1993) son maduras e incluso se encuentran estandarizadas. Sin embargo, en química (McKenna, 1991) e ingeniería mecánica, se carece de una interpretación específica.
En ingeniería mecánica, el enfoque primordial de las investigaciones en ingeniería inversa consiste en el desarrollo de modelos CAD de productos (representaciones geométricas en dos o tres dimensiones realizadas en sistemas CAD). Otras líneas de trabajo se abocan al reúso de diseños y al rediseño.

Este artículo presenta las principales líneas de investigación sobre ingeniería inversa que se desarrollan en el ámbito de la ingeniería mecánica a nivel mundial. La realización de modelos $C A D$, que es la línea de investigación más explorada, es la primera en ser mostrada. Puntos de vista sobre el término, aplicaciones y tecnología empleada son señaladas en la segunda sección. En la tercera, se incluye la investigación que hace uso de modelos de información computacionales para el desarrollo de modelos CAD. Las secciones siguientes presentan métodos de ingeniería inversa para la mejora de diseños, considerando no sólo la geometría sino otros 
aspectos de los productos (función, proceso de diseño e información sobre su ciclo de vida). Finalmente, se presentan las conclusiones.

\section{Investigación en ingeniería inversa basada en modelos CAD}

En ingeniería mecánica, ingeniería inversa no tiene una definición única. Comúnmente, ingeniería inversa denota el proceso por el cual una parte (componente) existente, es recreada o reproducida sin contar con sus dibujos detallados (Bidanda et al., 1991). Otras interpretaciones son: la ingeniería inversa pretende la creación de modelos CAD de superficies, basados en la apariencia, más que en requerimientos ingenieriles; la ingeniería inversa consiste en la elaboración de dibujos de partes existentes cuando la información de diseño original no está disponible (Kwok y Eagle, 1991); la ingeniería inversa es una herramienta para actualizar la base de datos CAD de productos, cuando su diseño original ha sido modificado en producción (Yau et al., 1993). Es importante destacar, que aun cuando no todas las definiciones anteriores mencionan al desarrollo de representaciones $\mathrm{CAD}$, los procesos y aplicaciones discutidos por los autores citados coinciden en la elaboración de dicha representación como meta esencial.

Las definiciones dadas hasta ahora a la ingeniería inversa han dependido del objetivo que se pretende alcanzar al usarla. Por lo general, el objetivo es la extracción de datos geométricos de productos, para ingresarlos a sistemas CAD. Las representaciones CAD obtenidas de esta forma pueden tener diferentes usos:

Diseño de nuevos productos. El proceso de diseño de algunos productos inicia con prototipos o modelos físicos, como es el caso de los definidos por condiciones estéticas. Técnicas de ingeniería inversa son usadas para: a) representar al prototipo con un modelo de información computacional; b) mejorar u optimar el diseño con sistemas CAD; y c) fabricar un producto con equipo CAM usando el modelo CAD (Bidanda et al., 1991; Hosni y Ferreira, 1994).

Modificación de diseños existentes. Los sistemas CAE ofrecen muchas ventajas para modificar diseños, pero el modelo CAD del producto a mejorar es necesario y en muchos casos no se cuenta con él. Además, el proceso de diseño de varios productos incluye la prueba y modificación de un prototipo. En estos casos, se requiere del modelo CAD del prototipo modificado para finalizar el proceso de diseño o para hacer cambios al producto (Milroy et al., 1996; Abella y Daschbach, 1994).

Substitución de partes desgastadas o rotas. Cuando una parte única se desgasta o rompe y no se cuenta con sus planos, se puede manufacturar una copia usando su modelo CAD (Bidanda et al., 1991; Puntambekar et al., 1994).

Inspección industrial. Una alternativa para verificar partes mecánicas es comparar sus dimensiones físicas con su modelo CAD. Usando este método, cualquier variación puede ser fácilmente identificada (Bidanda et al., 1991; Hosni y Ferreira, 1994). La inspección de objetos largos es un caso especial de esta aplicación, pues con frecuencia, no es posible medir con precisión partes de grandes dimensiones haciendo uso de equipo tradicional. El modelo CAD de estos objetos puede ser obtenido por medio de reconstrucción de imágenes parciales y ser empleado para alterar el diseño, inspeccionarlo o documentarlo (Bidanda et al., 1991).

Documentación de diseños. Productos diseñados por medio de sistemas CAD son frecuentemente modificados durante la etapa de producción para ajustar detalles o hacer mejoras; así, el modelo CAD debe ser actualizado para que corresponda al resultado final (Puntambekar et al., 1994; Huang y Motavalli, 1994; Abella y Daschbach, 1994).

Desarrollo de información para la manufactura. A partir de la información extraída de un producto, es posible generar la trayectoria de herramientas en control numérico. Ésta puede ser usada en producción (Kim y Ko, 1994; Sobh y Owen, 1995; Huang y Motavalli, 1994) para definir moldes o dados (Hosni y Ferreira, 1994) o para producir modelos mediante técnicas de manufactura rápida (Aranson, 1996; Lin y Chen, 1997).

Análisis de mercado. La realización de modelos CAD implica un análisis detallado del producto. Si se hace el análisis de varios diseños, de diferentes fabricantes, es posible, mediante estudios específicos, identificar las mejores ideas y tecnologías usadas por otras compañías (Aranson, 1996). Esto es útil para predecir tendencias tecnológicas futuras (Kokubo, 1993).

Varias técnicas y procedimientos de la ingeniería inversa, los cuales incluyen herramientas computacionales altamente especializadas, han sido desarrollados para casos muy particulares; por ejemplo, el software para aplicar ingeniería inversa a engranes (como lo muestra Kotlyar, 1991). En estas situaciones, la ingeniería inversa consiste en medir las características geométricas esenciales del espécimen para determinar con esta información el resto de las dimensiones de la pieza. Estas técnicas no pueden ser utilizadas en otro tipo de productos o componentes.

Tecnología de soporte para la ingeniería inversa basada en modelos CAD

Aranson (1996) ha descrito algunos productos comerciales para realizar ingeniería inversa. Estos productos incluyen 
equipo para inspección, paquetería de análisis y máquinas de manufactura rápida de prototipos. A pesar de su variedad y versatilidad, estos productos aún presentan varias limitaciones para generar modelos CAD.

Para construir el modelo CAD de un componente cuando se aplica ingeniería inversa, se deben realizar tres actividades principales: a) extraer información geométrica (dimensiones, forma); b) interpretar esta información y, a partir de ella, desarrollar modelos geométricos (relacionar superficies y volúmenes); y c) convertir esta interpretación para que pueda ser usada por un sistema CAD. El desarrollo de las primeras dos actividades es frecuentemente llamado digitalización.

La información geométrica de un producto o de alguno de sus componentes puede ser extraída mediante dos procedimientos que se pueden clasificar en métodos de contacto y métodos sin contacto. Los métodos de contacto incluyen a los instrumentos y técnicas de medición convencionales, entre los que se encuentran máquinas de medición por coordenadas y digitalización electromagnética y sónica (Bidanda y Hosni, 1994). Cámaras de vídeo, escáners láser y otras técnicas que usan luz para medir, corresponden al grupo de métodos sin contacto. La figura 1 presenta una configuración elemental de un sistema de ingeniería inversa.

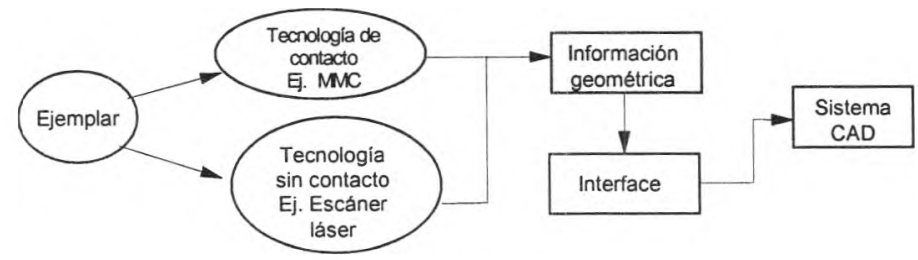

Figura 1. Configuración típica de un sistema de ingenieria inversa (Bidanda et al., 1991)

El análisis de la información geométrica obtenida de una pieza es, actualmente, un tema de investigación importante. La definición de la geometría real, a partir de puntos provistos por métodos de inspección, requiere dos actividades principales. La primera, es hacer una interpretación matemática para definir formas y estimar información faltante. Entre las técnicas de interpretación se encuentran: superficies algebraicas, superficies paramétricas, métodos de ajuste de superficies y robustez de ajustes. La segunda actividad requerida para analizar los datos de piezas digitalizadas es la modificación de la información para darle un formato apropiado para un sistema CAD (imágenes en formato ráster o vectorizadas). El trabajo en esta área es notificado por Sarkar y Menq (1991), Milroy et al. (1996), y Song y Kim (1997) entre otros.

Los sistemas CAD son una tecnología fundamental para la realización de ingeniería inversa basada en la digitalización; sin embargo, dichos sistemas no son investigados como parte de la ingeniería inversa. Actualmente, el desarrollo de herramientas CAD en ingeniería inversa es considerado sólo para generar modelos de productos muy específicos, los cuales involucran procedimientos altamente especializados, como es el caso de engranes (Kotlyar, 1991).

Sistemas de ingeniería inversa que emplean tecnología de contacto

La técnica de ingeniería inversa más común emplea equipo de medición por coordenadas para obtener la información geométrica de partes y transmitirla a un sistema CAD/CAM. El modelo CAD (geometría) es producido con el sistema CAD/CAM, que hace posible la generación de la trayectoria de corte para fabricar dicha parte (Oh et al., 1989).

Varios investigadores informan sobre el uso de la configuración "máquina de medición de coordenadas (interfaz) sistema CAD/CAM" para aplicar ingeniería inversa; algunos de ellos son Gupta y Sagar (1993), Hansen et al., (1993), Kwok y Eagle (1991), Yau et al., (1993), Kalra et al., (1991), Abella y Daschbach (1994). El empleo de esta configuración presenta dos problemas básicos. Uno, es la falta de un protocolo estándar de intercambio de información entre el equipo de medición y los sistemas CAD/CAM, lo cual dificulta la transferencia de la misma.

El otro, está relacionado con la exactitud de los datos producidos por las técnicas de medición. A este respecto, el reto ha sido generar un modelo CAD en tres dimensiones, de la parte inspeccionada, el cual sea fiel al objeto real si se emplean métodos matemáticos que requieran mediciones de pocos puntos del producto.

Sistemas de ingeniería inversa que emplean tecnología sin contacto

Los métodos sin contacto tienen dos ventajas fundamentales: a) prescindir de la difícil simulación del sentido del tacto; y b) su velocidad en la adquisición de datos. Estos métodos usan cámaras de vídeo, escáners láser, el método Moire o la combinación de éstos; varían dependiendo del uso que dan a la luz o sonido, tipo y patrón de la luz empleada, y posición y número de instrumentos de captación. Las aplicaciones notificadas de estos métodos de ingeniería inversa han aumentado en años recientes (Evans, 1991; Hosni et al., 1993; Motavalli y Bidanda, 1994; Milroy et al., 1996; Huang y Motavalli, 1994; Sobh y Owen, 1995). Las respectivas tecnologías se encuentran aún en investigación pero los resultados obtenidos hasta ahora son bastante prometedores. Cabe señalar, además, que estos métodos han sido combinados con tecnologías de manufactura rápida (Hosni et al., 1993). 


\section{Sistemas de ingeniería inversa basados en modelos de productos}

Motavalli y Shamsaasef (1996) desarrollaron un sistema para ingeniería inversa que incluye tecnología de contacto y tecnología sin contacto, un módulo para el reconocimiento de características (features) e información proporcionada por el usuario para generar una representación computacional de componentes. La representación computacional, o modelo de producto, incluye: una representación de la pieza en un sistema CAD; datos básicos referentes a la manufactura del componente, como acabados superficiales, tolerancias y materiales; y datos sobre las herramientas básicas y procesos requeridos para fabricar la parte.

En primer instancia, se utiliza un sistema de visión para digitalizar una parte. La imagen es procesada y sus fronteras son extraídas. Estas fronteras son empleadas para realizar un modelo CAD en dos dimensiones. El modelo es usado para generar un programa de control numérico, el cual conduce al palpador de una máquina de medición por coordenadas para capturar datos precisos de la superficie del objeto.

El proceso descrito es repetido varias veces desde diversos puntos de referencia. Al final, los datos son manejados para obtener un dibujo CAD en dos dimensiones.

El módulo para reconocimiento de características del sistema propuesto por Motavalli y Shamsaasef (1996) identifica características de forma, comparando las vistas ortogonales producidas por el modelo CAD con las proyecciones correspondientes de elementos geométricos pertenecientes a una librería almacenada en el sistema.

El modelo del producto está implantado en una base de datos orientada a objetos, y es capaz de almacenar y proveer la información requerida por todos los módulos que ejecutan las funciones del sistema (figura 2).

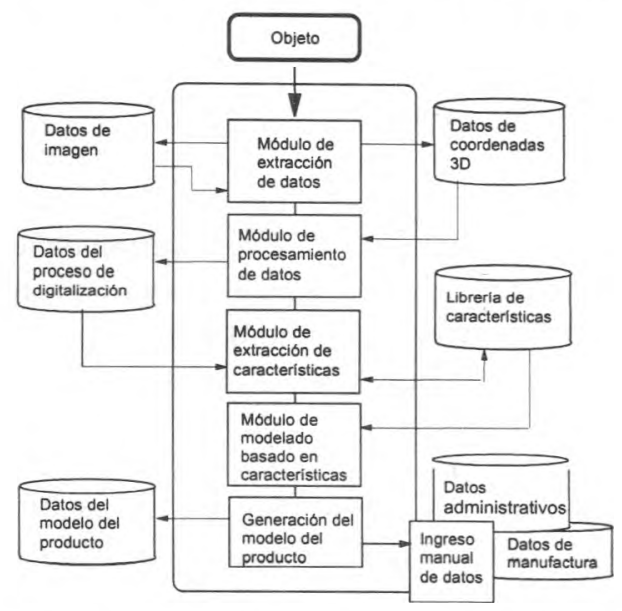

Figura 2. Base de datos para ingeniería inversa (Motavalli y Shamsaasef, 1996)

\section{Otros puntos de vista sobre ingeniería inversa}

El método de ingeniería inversa de Rekoff

Para Rekoff (1984), ingeniería inversa "es el acto de crear un conjunto de especificaciones para sistemas hardware por medio del análisis y dimensionamiento de un espécimen (o de una colección de especímenes)". En esta definición, un sistema hardware puede ser un sistema mecánico, eléctrico, electrónico o de software. Rekoff enfatiza la necesidad de entender el funcionamiento de un producto o parte cuando está dañado y se desarrolla una copia para ser utilizada como refacción. En este caso, extraer la geometría y dimensiones no es suficiente porque esta información no corresponderá a las propiedades originales de dicha parte, antes de desgastarse por el uso.

En su método (figura 3), Rekoff sugiere elaborar una descomposición jerárquica de un producto para después analizar dos de sus elementos en forma simultánea: artículo y elemento. El método puede ser aplicado a cualquier nivel de la descomposición.

Dos especificaciones son definidas para describir un artículo: a) la funcional, que detalla cómo un artículo y sus elementos trabajan e interactúan entre ellos; y b) la dimensional, que establece las dimensiones de la parte, su material y cómo es transformado durante la manufactura, los valores de parámetros y sus tolerancias, además de cómo es ensamblado el artículo.

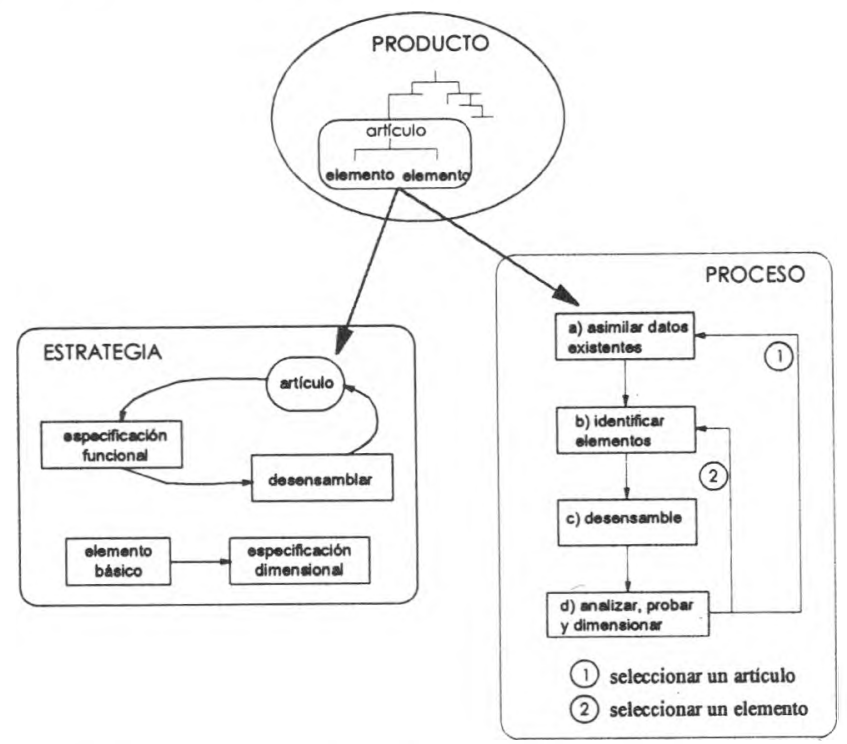

Figura 3. Método de Rekoff para ingeniería inversa

De acuerdo con Rekoff, la estrategia general de la ingeniería inversa es tomar un producto, establecer una especificación funcional inicial, desensamblar el componente cuya función ha sido claramente entendida, realizar la 
correspondiente especificación funcional, desensamblar de nuevo y repetir el proceso nuevamente. Esta secuencia de pasos es repetida hasta lograr un buen entendimiento de todos los componentes. Después de esto, cada elemento es aislado y medido para completar su especificación dimensional. De este modo, y en resumen, el método de ingeniería inversa consiste en una secuencia de cinco pasos: 1) asimilar datos existentes; 2) identificar elementos; 3) desensamblar; 4) analizar; y 5) probar y dimensionar. Estos pasos son ejecutados en forma cíclica como se ilustra en la figura 3.

\section{Ingeniería inversa usando la secuencia de diseño}

Kim y Bekey (1994) presentan un proceso de rediseño basado en la secuencia de diseño. Para ellos, ingeniería inversa es la "actividad de: a) adquirir o inferir el proceso y la secuencia de diseño usados para crear un producto dado; y b) emplear el conocimiento inferido para recrear el diseño o rediseñar un producto".

Estos autores sostienen que si un producto ha de ser rediseñado pero no se tiene acceso a su proceso de diseño, entonces, la ingeniería inversa puede ser usada para asumir uno. El proceso de diseño obtenido es empleado entonces para rediseñar por medio de una "re-ejecución y modificación" (Mostow, 1989).

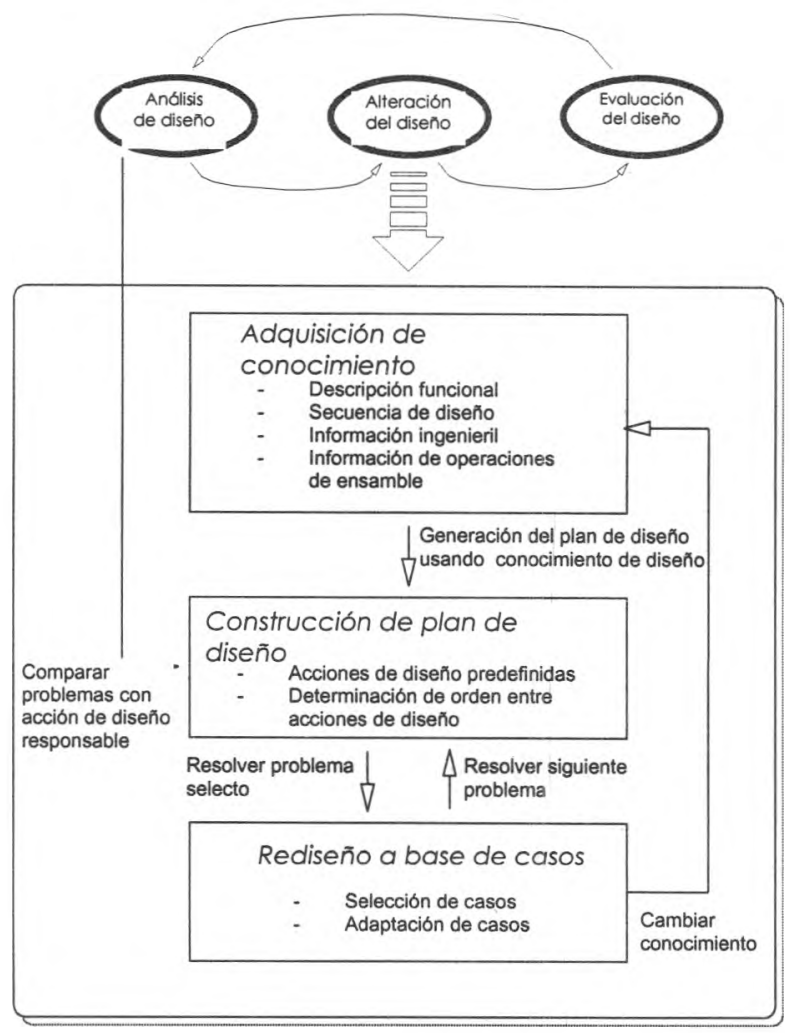

$\longrightarrow$ Tráfico pesado $\longrightarrow$ Tráfico ligero

Figura 4. Arquitectura de REV-ENGE (Kim y Bekey, 1994)
Kim y Bekey proponen un sistema para asistir el rediseño para ensamble, capaz de aplicar ingeniería inversa a componentes, creando un proceso de diseño basado en una secuencia de diseño generada. El sistema REV-ENGE (figura 4) opera bajo el supuesto de un modelo de diseño de tres etapas: análisis, alteración y evaluación. Primeramente, REV-ENGE modela la alteración del diseño como un ciclo de tres actividades principales: adquisición de conocimiento, construcción de un proceso de diseño y modificación del diseño.

Para resolver alguna falla de un producto por medio de su rediseño usando REV-ENGE, se proporciona al sistema la descripción del diseño, incluyendo su especificación. El módulo de adquisición de conocimiento del producto adquiere interactivamente la secuencia de diseño basándose en la metodología de diseño para ensamble. Posteriormente, se construye un proceso de diseño con ella, de tal forma que cada actividad es justificada por una o más secuencias de diseño.

La falla, o fallas, que dieron motivo al rediseño son identificadas con porciones del proceso de diseño durante las cuales pudieron originarse. Luego, el proceso de diseño es nuevamente ejecutado, modificando las decisiones, y en consecuencia, las acciones o porciones del proceso relacionadas con la falla, asistiéndose de la experiencia de diseños previos a través de un método basado en casos.

Kim y Bekey aclaran que la validez del procedimiento descrito se fundamenta en que el producto rediseñado será similar al original, y que sólo ha sido probado considerando un proceso de diseño basado en el método de diseño para ensamble. La modificación del diseño es un proceso iterativo debido a que en cada ocasión en que se efectúa un cambio al diseño, es necesario verificar si algunas especificaciones han sido violadas o se han creado nuevos problemas.

\section{Ingeniería inversa en el contexto de la ingeniería concurrente}

Borja (1997) establece que la ingeniería inversa es el proceso de diseñar un substituto que reemplace de forma aceptable a un producto o parte. En este caso, ingeniería inversa es un caso particular de rediseño fundamentado en diversos aspectos del producto original y en el análisis de un ejemplar, y se aplica cuando el proceso de diseño o la documentación original no estar disponible.

El proceso propuesto (figura 5) incluye tres etapas: a) captura y análisis de información del producto original; b) definición del concepto (funcionalidad) y geometría de un substituto; y c) especificación del proceso de manufactura. La primera etapa incluye la recolección del conocimiento de diseño e información del producto cuyo substituto es requerido, y la elaboración de una especificación inicial del substituto. 


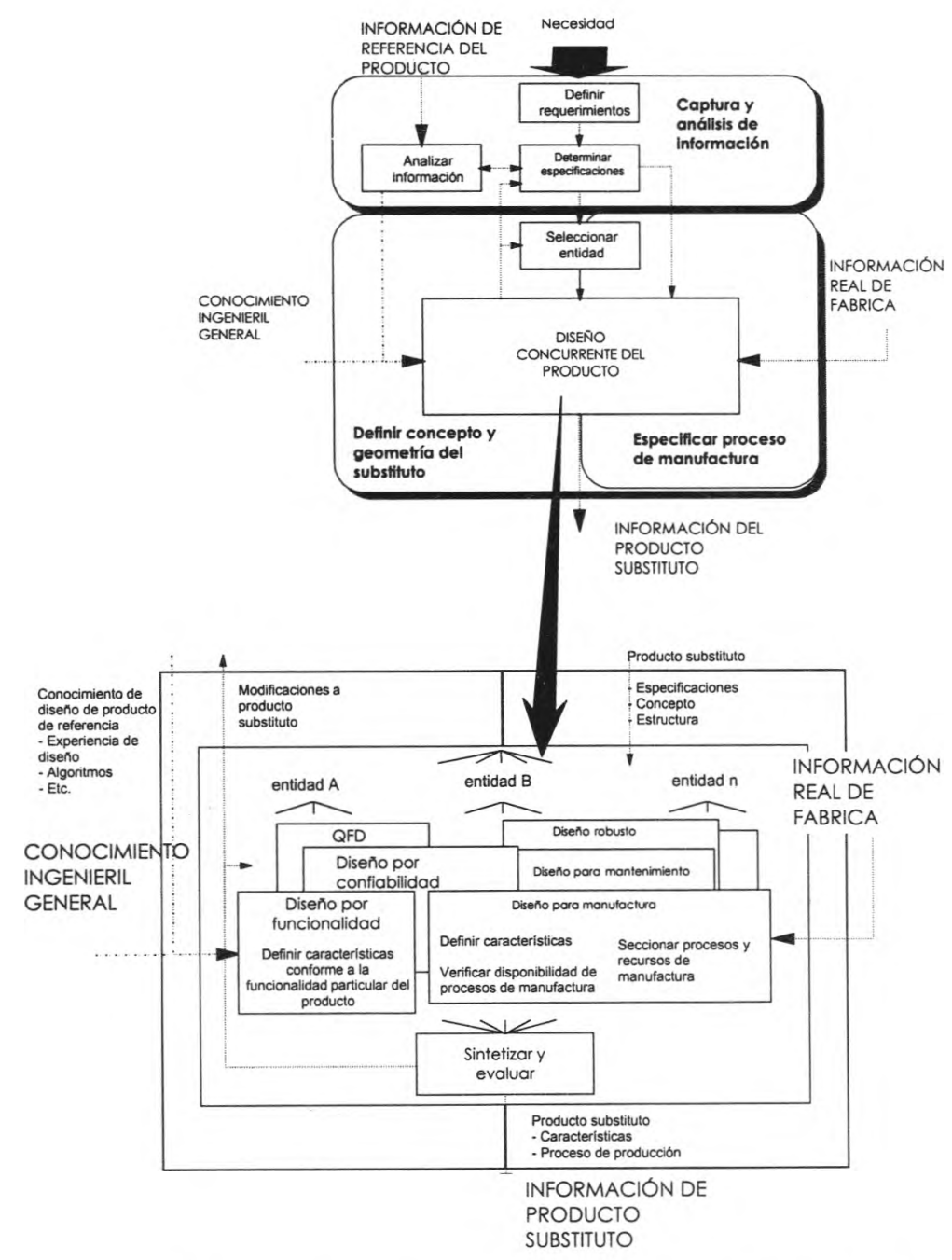

Figura 5. El proceso de ingeniería inversa (Borja, 1997)

La segunda etapa del proceso consiste en la evaluación y modificación de la especificación del substituto basándose en conocimiento del producto original y en información de las capacidades de manufactura disponibles. El proceso de ingeniería inversa termina con la selección de los recursos y procesos de manufactura necesarios para producir el substituto.

Es importante destacar que las últimas dos etapas se desarrollan en forma simultánea y, por tanto, siguen los principios de la ingeniería concurrente: varias actividades se realizan en forma simultánea, se atacan diferentes aspectos del producto y se toman decisiones sobre el proceso productivo mientras se realiza el diseño (figura 5).

Borja (1997) también propone un sistema CAE basado en modelos de información para auxiliar la aplicación del proceso descrito. En este sistema se destacan dos bases de datos que centralizan la información referente a productos y manufactura, así como un programa que emplea esta información para auxiliar el diseño para manufactura.

\section{Conclusiones}

Se han presentado diferentes enfoques sobre la ingeniería inversa en el contexto de la ingeniería mecánica; a partir de las referencias hechas, es fácil concluir que la investigación sobre el tema se centra, principalmente, en la digitalización y generación de modelos CAD, siendo las principales aplicaciones: la realización de piezas de refacción y la documentación. Considerando el trabajo de Motavalli y Shamsaasef (1996), se puede apreciar la tendencia futura del uso de modelos de información y la consideración de información referente a la manufactura en sistemas que auxilian al desarrollo de sistemas CAD. En este sentido, un número cada vez mayor de investigadores está adoptando el uso de la norma internacional de representación e intercambio de datos de productos desarrollada por la organización internacional de estándares, comúnmente conocida como STEP (ISO10303-1), que ofrece la ventaja de facilitar la realización de modelos de información y la transferencia de información a sistemas CAD/CAM. 
Por otro lado, también se presentaron las visiones más amplias sobre ingeniería inversa, que pueden incluir a las anteriores. Tanto Rekoff (1984) como Kim y Bekey (1994), y Borja (1997) destacan la importancia de analizar productos en forma integral, y no limitarse a aspectos geométricos y dimensionales. Se enfatiza como punto principal el conocimiento del producto que incluye su funcionalidad y proceso de diseño. Estas propuestas sobre ingeniería inversa, la plantean como una técnica de rediseño que permite la mejora tanto de productos como de componentes.

En opinión de los autores, es necesario promover una idea más completa acerca de la ingeniería inversa, para explotar sus posibilidades de adaptar productos a contextos específicos - Borja 1997, considera recursos de manufactura particulares - y mejorarlos (Kim y Bekey, 1994 y Borja, 1997). De esta forma, las aplicaciones de la ingeniería inversa pueden extenderse a la asimilación de tecnología y a la predicción de tendencias tecnológicas futuras.

\section{Agradecimientos}

Este artículo está basado en una investigación patrocinada por la UNAM y realizada en Loughborough University, Gran Bretaña. Los autores desean agradecer a los miembros del grupo de investigación de CAE del Departamento de Manufactura de Loughborough University, sus valiosos comentarios y apoyo.

\section{Referencias}

Abella R. y Daschbach J.M. (1994). Reverse Engineering Industrial Applications. Computers Ind. Engng, Vol. 26, No.2, 381-385.

Aranson R.B. (1996). Forward Thinkers Take to Reverse Engineering, Manufacturing Engineering, November, 34-44.

Bidanda B. y Hosni Y.A. (1994). Reverse Engineering and its Relevance to Industrial Engineering: a Critical Review, Computers Ind. Engng, Vol. 26, No. 2, 343-348.

Bidanda B., Motavalli S. y Harding K. (1991). Reverse Engineering: an Evaluation of Prospective Non-contact Technologies and Applications in Manufacturing Systems, Int. J. Computer Integrated Manufacturing, Vol. 4, No. 3, 145-156.

Borja V. (1997). Redesign Supported by Data Models with Particular Reference to Reverse Engineering. PhD Thesis of the Department of Manufacturing Engineering, Loughborough University.
Evans M.S. (1991). Robotics Based Part Inspection and Reverse Engineering System, en: Proceedings 36th Conference on Remote Systems Technology, pp. 189-193.

Gupta V.K. y Sagar R. (1993). A PC-Based System Integrating $\mathrm{CMM}$ and $\mathrm{CAD}$ for Automated Inspection and Reverse Engineering, The International Journal of Advanced Manufacturing Technology, Vol. 8, 305-310.

Hansen F., Pavlakos E., Hoffman E., Kanade T., Reddy R. y Wright P. (1993). PARES: A Prototyping and Reverse Engineering System for Mechanical Parts-on-Demand on the National Network, Journal of Manufacturing Systems, Vol. 12, No. 4, 269-281.

Hosni Y. y Ferreira L. (1994). Laser Based System for Reverse Engineering, Computers Ind. Engng, Vol. 26, No.2, 387-394.

Hosni Y., Ferreira, L. y Burjanroppa R. (1993). Rapid Prototyping Through Reverse Engineering, en: 2nd Industrial Engineering Research Conference Proceedings, pp. 420-424.

Huang C.N. y Motavalli S. (1994). Reverse Engineering of Planar Parts Using Machine Vision. Computers Ind. Engng, Vol. 26, No.2, 369-379.

ISO 10303-1 Industrial Automation Systems (1994). Product Data Representation and Exchange. Part 1: Overview and Fundamental Principles.

Kalra V., Sheth P.N. y Richards L.G. (1991). Reverse Engineering of Mechanical Components within a Computer-aided, Manufacturing Environment. Journal of Engineering Design, Vol. 2, No. 3, 219-230. Kim G.J. y Bekey G.A. (1994). Artificial Intelligence in Design '94. Kluwer Academic Publisher, Netherlands.

Kim K. y Ko B. (1994). Generating Cartesian NC Tool Paths for Sculptured Surface Manufacture. Computers Ind. Engng, Vol. 26, No.2, 356-367.

Kokubo A. (1993). Competitive Intelligence, IEEE Spectrum, Vol.30, Iss. 8, August, 44-46.

Kotlyar Y. (1991). Gear Fundamentals Reverse Engineering. Gear Technology, Vol. 8, Part 4, 34-38.

Kwok W.L. y Eagle P.J. (1991). Reverse Engineering: Extracting CAD Data from Existing Parts. Mechanical Engineering, Vol. 13, Iss. 3, March, 52-55.

Lin G.C.I. y Chen L.C. (1997). An Automatic Reverse Engineering Approach for Rapid Prototyping Manufacturing, en: Proceedings of the International Conference on Manufacturing Automation. Vol. 2, ICMA '97, April pp. 28-30, Hong Kong, pp. 903-908.

McKenna J.F. (1991). Shuter Inc. Gives New Meaning to Etc. Industrial Week, Vol. 240, Iss: 3, February 4, 44-48, USA.

Milroy M.J., Weir D.J., Bradley C. y Vickers G.W. (1996).

Reverse Engineering Employing a 3D Laser Scanner: a 
case Study. The International Journal of Advanced Manufacturing Technology, No. 12, 111-121.

Motavalli S. y Bidanda B. (1994). Modular Software Development for Digitilizing Systems data Analysis in Reverse Engineering Applications: case of Concentric Rotational Parts. Computers Ind. Engng, Vol. 26 , No.2, 395-410.

Motavalli S. y Shamsaasef R. (1996). Object-oriented Modelling of a Feature-based Reverse Engineering System. Int. J. Computer Integrated Manufacturing, Vol. 9, No. 5, 354-368.

Mostow J. (1989). Artificial Intelligence.

Oh K.H., Abella R.J. y Daschbach J.M. (1989). CMM Application in Reverse Engineering. Integrating CNN with CAD/CAM for Existing Parts Without Drawings. SME Technical Paper (Series) MS 89 19, Ohio

Puntambekar N.V., Jablokow A.G. y Sommer III, H.J. (1994). Unified review of 3D Model Generation for Reverse Engineering. Computer Integrated Manufacturing Systems, Vol. 7, No.4, 259-268.

Rauch J.G. (1993). The Law on Reverse Engineering, IEEE Spectrum, Vol. 30, Iss. 8, August, 47-48.
Rekoff M.G. (1984). Method for Reverse Engineering, Instrumentation in the Aerospace Industry, Vol. 30, 619-625.

Sarkar B. y Menq C.H. (1991). Smooth-Surface Approximation and Reverse Engineering. Computer-Aided Design, Vol. 23, Num. 9, 623-628.

Sobh T. y Owen J. (1995). A Sensing Strategy for the Reverse Engineering of Machined Parts. Journal of Intelligent and Robotic Systems, No.14, 323-340.

Song C.K. y Kim S.W. (1997). Reverse Engineering: Autonomous Digitization of Free-Formed Surfaces on a CNC Coordinate Measuring Machine. Int. J. Mach. tools Manufact, Vol.37, No.7, 1041-1051.

West R. (1993). Reverse Engineering an Overview, Information Systems Engineering Library, CCTA. November, London.

Yau H.T., Haque S. y Menq C.H. (1993). Reverse Engineering in the Design of Engine Intake and Exhaust Ports, American Society of Mechanical Engineers. Production Engineering Division (Publication) PED: Manufacturing Science and Engineering, ASME, New York, USA.

\section{Semblanza de los autores}

Vicente Borja-Ramirez. Graduado como ingeniero mecánico electricista (1989) y maestro en ingeniería mecánica (1992) en la Facultad de Ingeniería (FI), UNAM. Realizó su investigación doctoral en Loughborough University sobre ingeniería asistida por computadora y obtuvo el grado en 1997. Es profesor del Departamento de Ingeniería Mecánica de la Facultad desde 1990, imparte cátedra y participa en proyectos vinculados con empresas en el área de diseño mecánico. Ha sido jefe del Centro de Diseño y Manufactura, así como secretario técnico de la Coordinación de la Investigación Aplicada de la misma Facultad. Actualmente, es jefe de la Sección de Mecánica de la División de Estudios de Posgrado de la FI, UNAM.

Robert Bell. Se graduó como ingeniero electricista en 1994 y como maestro en tecnología textil en 1996, en el UMIST, Gran Bretaña. Obtuvo el grado de doctor en la misma Universidad en 1975. Fue nombrado profesor de tecnología de manufactura en Loughbo. rough University, Gran Bretaña, en 1978. Ha establecido la línea de investigación en sistemas de manufactura flexible, particularmente en métodos de modelado para el diseño de celdas. Actualmente, dirige proyectos en las áreas de modelos de productos y manufactura en CIM, conceptos sobre modelado de fábricas, y sistemas de administración de herramientas.

Marcelo López-Parra. Egresado de la carrera de ingeniería mecánica y eléctrica de la Facultad de Ingeniería, UNAM. Realizó la maes. tría en ingeniería mecánica en la misma Facultad y la maestría en robótica industrial en Cranfield University, Gran Bretaña. Esta última institución le otorgó el grado de doctor en ingeniería mecánica. Actualmente, es jefe del Centro de Diseño y Manufactura de la FI, UNAM.

Saúl D. Santillán-Gutiérrez. Obtuvo el grado de ingeniero en la FI, UNAM en 1988 y, en 1992, de maestro en ingeniería, en la misma Facultad. El grado de doctor lo obtuvo recientemente en Loughborough University, Inglaterra. Es profesor en el Centro de Diseño y Manufactura. Ha sido postulado tres veces para el premio Universidad Nacional para Jóvenes Investigadores entre 1992 y 1995.

Leopoldo A. González-González. Obtuvo el título de ingeniero mecánico electricista en 1989 y el grado de maestro en ingeniería en 1992, ambos con mención honorífica, en la FI, UNAM. Fue candidato a investigador nacional de 1992 a 1995 . Ha obtenido en dos ocasiones la cátedra especial "Javier Barros Sierra" y ha sido postulado tres veces al premio Universidad Nacional para Jóvenes Investigadores. Es profesor de carrera desde 1991, y de 1993 a 1996, fue el jefe del Centro de Diseño y Manufactura de la FI, UNAM, donde fue responsable de la contratación y ejecución de proyectos de desarrollo e innovación tecnológica. Actualmente, funge como consultor del CONACyT para la evaluación de proyectos de desarrollo tecnológico. 